\title{
HYPERTENSION AND UNILATERAL RENAL DISEASE
}

\author{
By S. B. Karani, M.B., B.S., M.R.C.P., D.P.H. \\ Senior Resident Physician, St. Nicho'as' Hospital (L.C.C.); Late Specialist Physician, R.A.M.C.
}

Richard Bright in his classical work published in 1836 , drew attention to the thickening of the renal arteries, with concomitant cardiac hypertrophy to be found in cases of chronic interstitial nephritis. These important facts were therefore known prior to the use of sphygmomanometer in clinical medicine, and since then considerable experimental work has been done to elucidate the relationship between hypertension and renal disease. In recent years special importance has been attributed to the role of renal ischaemia, particularly of a single kidney, in the production of hypertension.

Graefi and Page (1940) have performed interesting experiments on dogs. By surrounding one kidney with Cellophane they produced perinephritis with hypertension persisting for as long as 14 months; the hypertension subsided after removal of the diseased kidney. The histological findings were cortico-tubular atrophy with interstitial and perirenal fibrosis. Several writers, mostly in America have reported cases amongst children and young adults of hypertension associated with unilateral renal disease. Butler (1937) reports I5 cases of children who had pyelonephritis and and hypertension for a period of years before appreciable impairment of renal function occurred. He reports two instances in which removal of one pyelonephritic kidney was followed by return to normal of an elevated blood pressure. Kennedy, Barker and Walters (194I, 1945) report a case of unilateral atrophic pyelonephritis in a girl of seven years, with severe hypertension and angiospastic retinitis. Both the conditions cleared up after nephrectomy and her blood pressure remained normal throughout the five years after operation.

However, other writers have published results which show that nephrectomy fails to lower the elevated blood pressure and Boyd
(1942) does not regard the removal of the diseased kidney as a permanent cure. $\mathrm{He}$ warns us that the other kidney may be found affected after a number of years and he describes a case where the second kidney was found abnormal after ten years. Recently Robertson (1946) showed a case of hypertensive retinopathy in a girl of Io years II months, where both the kidneys were affected. Following bilateral adrenalectomy and right and left sympathectomy there was clinical improvement and the girl was alive and better three months after the operation.

The following is a report of a case of unilateral megalo-ureter and pyelonephritis with hypertension in a boy aged 13 years.

G.W. attended our casualty department on $30 / 12 / 46$ complaining of frontal headache and pain in the right elbow following an accident two days previously when he was knocked down by a cyclist. On clinical examination nothing abnormal was found (blood pressure was not recorded). The skiagram of skull, chest and right elbow revealed no bony injury. However, he was admitted for observation.

For the next three days except for an evening rise of temperature $\left(99^{\circ}-100^{\circ} \mathrm{F}\right.$.) nothing abnormal was noted. On the fourth day I was first asked to see him as his headache was more severe and he complained of spasmodic muscular pains in both the thighs and the arms. $\mathrm{He}$ refused all food, but there was neither nausea nor vomiting. He appeared ill and resented light. There was no rash nor any signs either of increased intracranial tension or meningeal irritation. His temperature was $100^{\circ} \mathrm{F}$. and the tongue was coated and moist. Nothing abnormal was found in chest, heart or abdomen. Blood pressure was 200/120 in both the brachial arteries, and his pulse was rapid (rate 130), regular and of high tension. Whilst recording the B.P. he complained of 
severe pain in the forearm which appeared flushed when the pressure was raised to only I00 $\mathrm{mm}$. The pain and the flushing immediately subsided when the pressure was released.

The following investigations were carried out :-

Urine : 24 hour urinary output was proportional to fluid intake, and the specific gravity of different samples varied from roro to IoI8. It was clear, protein + , centrifugal deposit showed a few pus cells and red blood cells, and very occasional epithelial cells. Gram stain: No organism seen. Ziehl Neelsen: No T.B. seen. Culture: Sterile. Blood count: Total W.B.C., I7,000 per $\mathrm{cmm}$. Neutrophils, 14,790. Lymphocytes, I,530 and Monocytes, 680 per $\mathrm{cmm}$. Blood culture : Sterile : Blood urea : $46 \mathrm{mg}$. per cent. C.S.F. : Normal. X-ray of chest, both arms and thighs : Normal.

The above findings led to the diagnosis of acute pyelonephritis. He was put on penicillin, I00,000 units stat and 30,000 units threehourly. Sulphathiozole by mouth was also started two days later as there was no clinical improvement. Following this double chemotherapy his condition began to improve. $\mathrm{He}$ became less toxic, his appetite returned and the headache and the muscular pain became less intense though his B.P. remained high. In all he was given 30 grammes of sulphathiozole and three million units of penicillin. Following this intensive course of chemotherapy, except for occasional nocturnal headaches which were relieved by ten grains of aspirin, he felt perfectly well. Further investigations gave the following results :-

Urine : Clear, sp. gr. ror 8. Protein : Trace, deposit showed occasional W.B.C.s and epithelial cells. Culture was sterile. Blood count: Hb. 88 per cent. Total W.B.C., I0,000 per cmm. Neutrophils, 8,200. Lymphocytes, I, 500 and Monocytes, 300 per $\mathrm{cmm}$. W.R. and Kahn: Negative. Blood urea : 30 mg. per cent. Serum chlorides : $350 \mathrm{mg}$. per cent. Serum calcium: $10.7 \mathrm{mg}$. per cent. Serum phosphate $5.4 \mathrm{mg}$. per cent. Plasma cholesterol : I $50 \mathrm{mg}$. per cent. Urea clearance test : 74 per cent. average normal. No satisfactory intravenous pyelogram or ureterogram was obtained, the vesiculogram was normal.
Retrograde pyelogram was done on 8/2/1947, no urine was obtained from the right ureter and the report on the urine from the left ureter was as follows :-Opalescent, protein trace, deposit showed occasional epithelial cells only. Ziehl Neelsen: No T.B. seen. Culture sterile. The pyelogram (Fig. I) showed enlarged left kidney with normal pelvic ureter, the right kidney was small with dilated pelvic ureter. This dilatation was thought to be due to congenital achalasis of the uretero-vesical orifice.

Nephro-ureterectomy was performed by Mr. J. Carver, F.R.C.S., on 22/2/1947. Blood pressure immediately following the operation was $140 / 80$ and on the next day it was $120 / 60$. Fig. 2 shows a graphic record of the blood pressure readings before and after the operation. He made an uninterrupted recovery following the operation, and at present he is in normal health.

The electrocardiogram (Fig. 3 ) is normal, the urine is free from protein and the centrifuged deposit shows no abnormality. The Urea Clearance Test is 68 per cent. average normal and the blood urea is $29 \mathrm{mg}$. per cent. Pathologist's report on the diseased kidney and the ureter is as follows (Figs. 4 and 5) :-

'Contracted hydronephrotic kidney; the pelvis shows severe chronic inflammation and the renal substance shows areas of fibrosis and round-celled infiltration, many tubules are dilated and atrophic and contain hyaline colloid material. Many glomeruli show varying degrees of fibrosis, and the smaller arterioles show intimal and medial thickening.

' Thickened fibrotic wall of lower end of excised ureter shows subepithelial chronic inflammatory infiltration.'

\section{Discussion}

Pathogenesis of megalo-ureter. It is suggested that congenital megalo-ureter can occur in either of the following ways :-

(i) Deficiency in the musculature of the ureteric walls.

(ii) Disturbed neuro-muscular mechanism.

(iii) Lack of normal inhibitory influences on the development of the ureter.

(iv) Obstruction of the lumen of the ureter by a congenital valvular defect.

This last ingenious explanation is put forward by Chawalla (1927). The adult kidney 


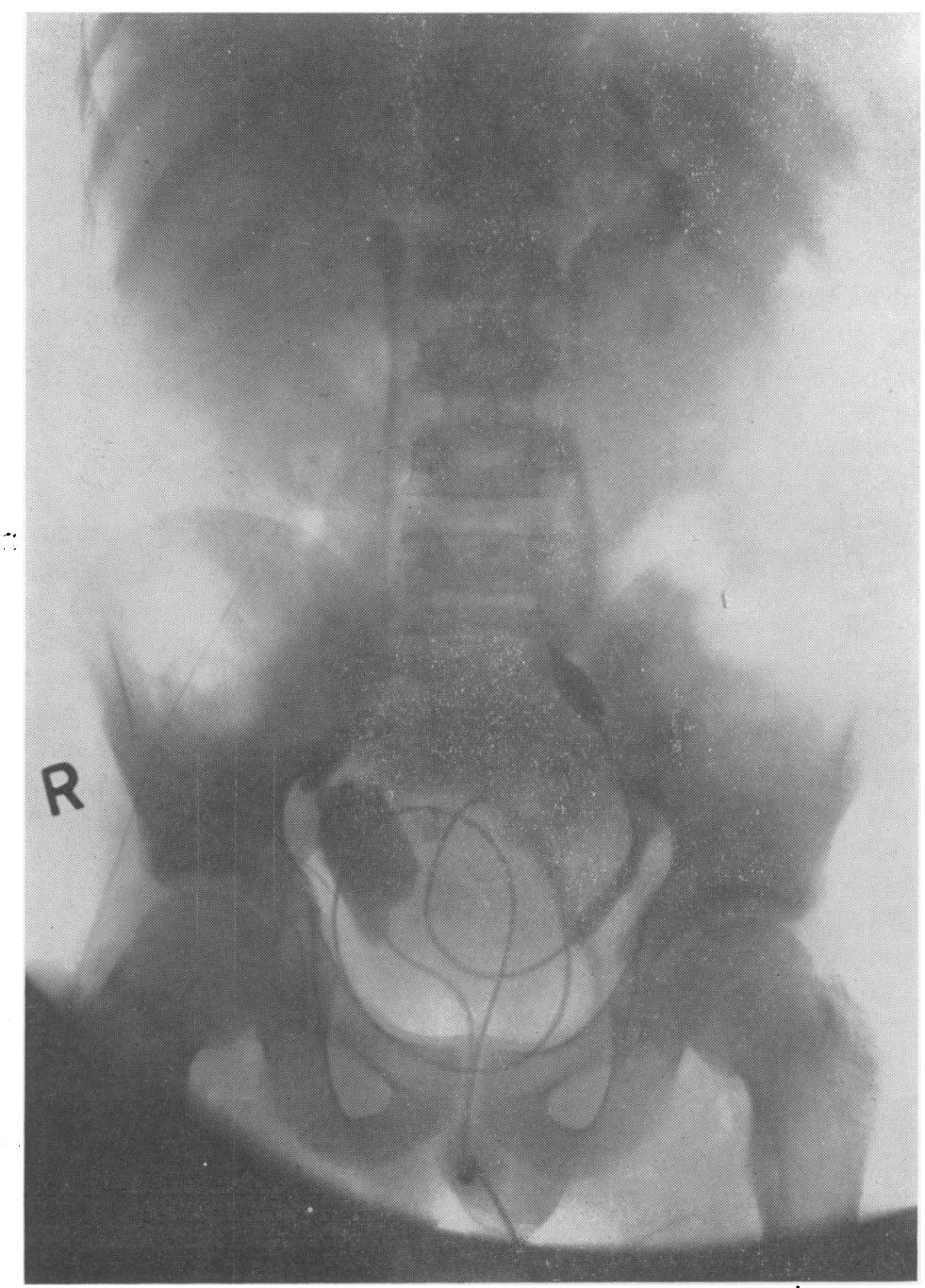

Fig. 1.- - Retrograde pyelogram showing enlarged left kidney with normal pelvic ureter; right kidney is small with dilated ureter. 


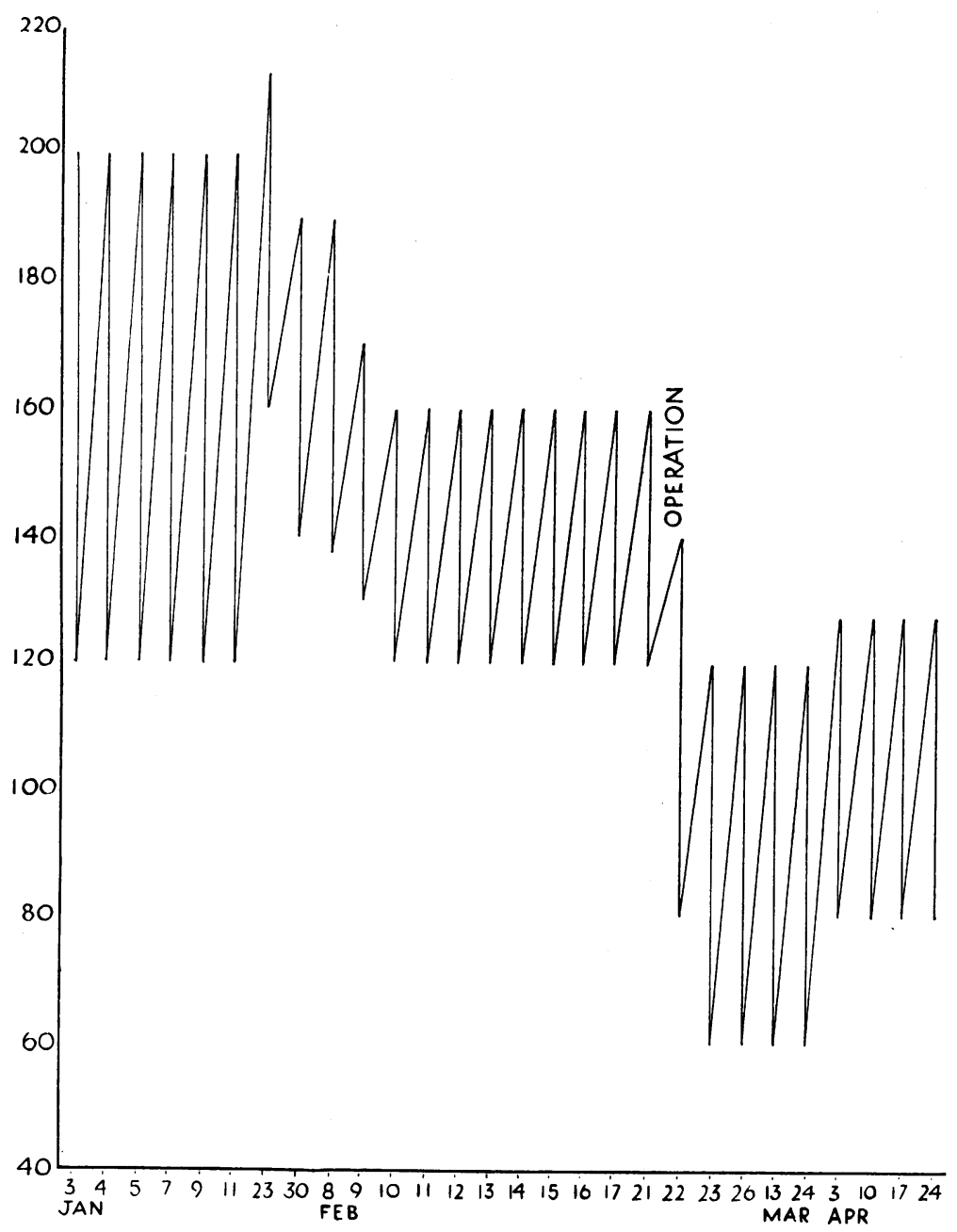

Fig. 2.-Graphic record of blood-pressure readings before and after the operation.

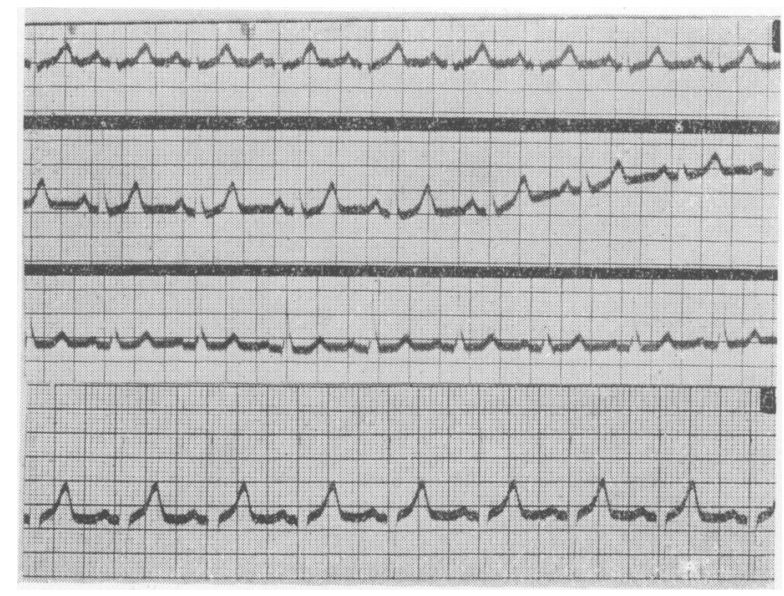

Fig. 3.-Normal E.C.G. tracing in all the leads after nephro-ureterectomy. 


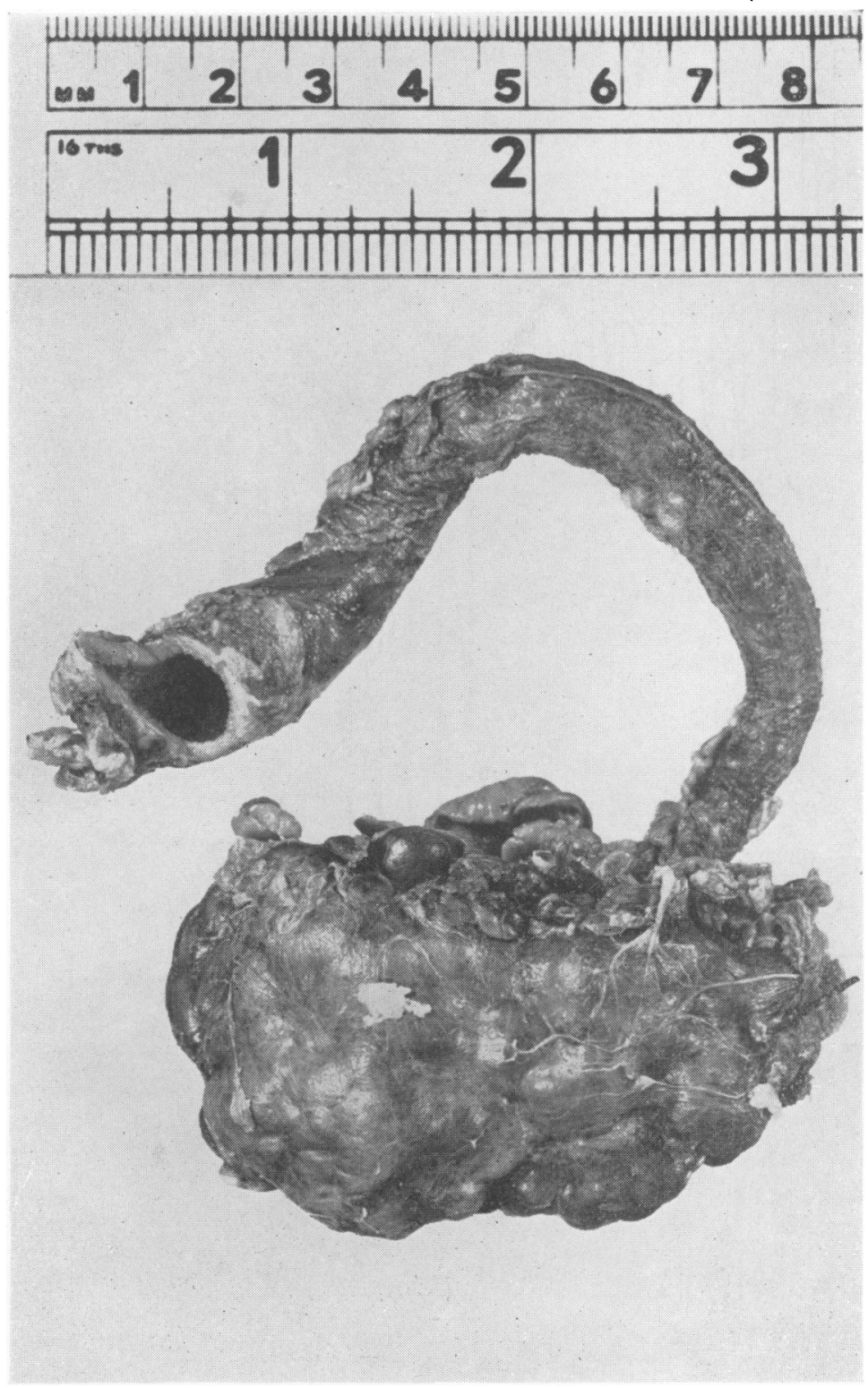

Fig. 4.-Small contracted kidney with dilated ureter. 


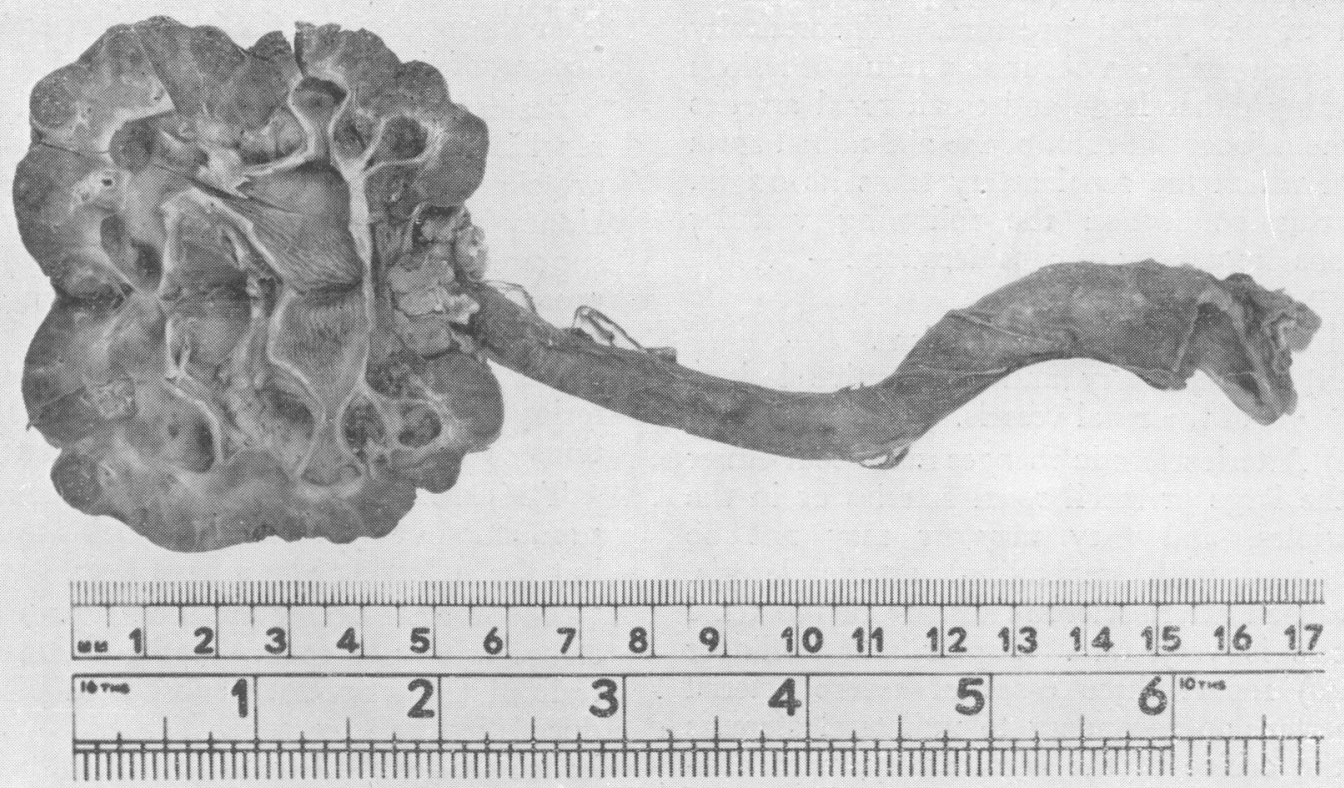

Fig. 5.-Cross-section of the atrophic kidney.

develops from the metanephros which is the early embryonic renal organ, whilst the ureter and the pelvis of the kidney develop from the Wolffian duct. When the human embryo is about $4 \mathrm{~mm}$. long the ureteral bud arises from the Wolffian duct and begins to develop until the embryo is $16 \mathrm{~mm}$. long. The bud then separates from the duct to form the ureter. The ureteral lumen as it separates from the duct is covered by a cellular membrane. After separation the ureter fuses with the pelvic portion of the kidney and whereas during fusion the membrane remains intact, it soon disintegrates because increasing pressure is exerted upon it owing to continual secretion from the embryonic kidney. The rupture of the membrane allows the fluid to escape. If for some reason the breakdown of the membrane does not occur - the fluid is thus retained and hydroureter and hydronephrosis results. Breakdown of the membrane at a later date can, however, result in normal development provided there is not a considerable delay.
If this occurs the dilatation recedes either slowly or partially and one may then find a normal ureteral orifice though still small for adequate drainage. Another possibility would be only partial disintegration of the membrane. This would result in the presence of either a congenital valve, diverticulum, stricture, or a ureterocele at or near the ureterovesical orifice. And so a megalo-ureter can result either from an obstruction or an imperfect drainage at the ureteral orifice. This occurrence of megalo-ureter predisposes to ascending infection giving rise to pyelonephritis of the insidious type ultimately causing atrophic contracted kidney. Boyd (I942) and others suggest that healed pyelonephritis caused either by a gradually ascending or blood-borne infection is a more common cause of an atrophic contracted kidney than hitherto assumed.

Renal ischaemia. Considerable interest has been taken by clinicians and pathologists in the role of renal ischaemia as a possible cause of 
hypertension in man after Goldblatt and his associates (1934) produced experimental renal ischaemia in animals causing a persistent elevation of the blood pressure. Theoretically renal ischaemia can occur as a result of lesions affecting either large and small renal arteries or the adjacent portion of the abdominal aorta from which the renal artery takes its origin. In this connection the following vascular lesions are worthy of consideration :-

(i) Arteriosclerosis.

(ii) Thrombosis and embolism.

(iii) Inflammatory and obliterative lesions of the renal vessels.

(i) Arteriosclerotic changes may occur either in the large or small renal arteries or in the arterioles, and they may or may not be associated with generalized arteriosclerosis. Localized arteriosclerotic disease of the renal vessels is very rare. Saphir and Ballinger (I940) report three cases of severe arterial hypertension secondary to unilateral stenosis of the orifice of the renal artery, with consequent ischaemia of one kidney. In two of these cases autopsy revealed unilateral malignant nephrosclerosis in the contralateral kidney. In generalized arteriosclerosis it remains doubtful whether hypertension is the cause of renal arteriosclerosis or its result. It is suggested that an initial spastic condition of the arterioles, perhaps due to sustained overactivity of the vegetative nervous system, is sufficient to produce hypertension. At the same time, however, prolonged spasticity of the ve els leads to arteriosclerosis which in turn contributes to a further rise of blood pressure. Persistent elevation of the blood pressure causes increasing spasticity of the vessels, thus closing the vicious circle. Therefore, according to this theory hypertension may be regarded as the cause and also the result of renal arteriosclerosis.

(ii) Thrombosis or embolism of the renal artery produces sudden onset of an acute abdominal catastrophy, associated with transient hypertension. At a later stage the degree of hypertension will depend on the extent of the renal damage as well as the previous condition of the kidney.

(iii) A review of literature gives very little information about cases of hypertension in connection with either inflammatory or ob- literative lesions like thrombo-angiitis obliterans and endarteritis obliterans of the renal arteries. It is believed that high blood pressure is an almost constant finding when periarteritis nodosa affects the renal vessels.

Besides the above lesions of the renal vessels, renal ischaemia can result from :- (a) Compression of the renal vessels at the hilum of the kidney by tumours either of the kidney or the suprarenal, e.g., Wilm's tumour; (b) Compression or distortion or angulation of the vascular pedicle due to a displaced kidney; (c) Changes in the renal parenchyma interfering with capillary circulation; (d) Affections of the abdominal aorta, e.g., aneurysm.

The exact role of the ischaemic kidney in the production of hypertension remains unknown, but the following views have been suggested.

The normal kidney produces a pressor substance known as renin, but its action is neutralized by antipressor substances also produced by healthy renal tissue. When the ischaemic renal tissue is in excess to healthy kidney substance, excess of renin is secreted. Experimental evidence in animals shows that renin has a direct pressor action causing generalized constriction of the arterioles. This action is enhanced by the circulating proteinglobulin. It is also suggested that cortin of the suprarenal influences the action of renin. Another view is that the ischaemic kidney produces a hormone-like substance which has a similar action to renin.

Another theory which is even more speculative is concerned with the importance of the so-called Juxta-Glomerulo apparatus for the pathogenesis of hypertension. German authors first described a group of cells of glomus type lying at the poles of the glomerulus (i.e., where the afferent and efferent arteries enter and leave the glomerular structure respectively) and called Polkissen, probably because of their situation and cushion-like appearance. Similar cells are said to be found in the inter-nephron spaces as well as in the walls of the afferent arterioles. Besides this, the juxta-glomerular apparatus comprises a central convoluted tubule lying adjacent to the glomerulus. At the point which is nearest to the glomerulus, the Macula Densa results from the characteristic arrangement of the fibrillar cells of the tubules. After special staining the cells of this apparatus 
can be demonstrated as either granular or afibrillar. Thus the juxta-glomerular apparatus is a conglomeration of polkissen, macula densa, afibrillar and granular cells. So far it has been difficult to demonstrate the granular content of the juxta-glomerular cells in human kidneys because of the difficulty to obtain an immediate and perfect fixation. It is suggested that this apparatus controls the circulation through the kidney substance and thus plays a part in the production of hypertension in kidney diseases. Boyd (1942) stated that he had found this apparatus normal in his cases of hypertension.

Congenital anomalies of the urinary tract predispose to urinary stasis, retention, infection and renal insufficiency. Moreover, various writers emphasize the importance of the initial occurrence of gastro-intestinal symptoms in their cases, probably due to the disturbance of renal-gastric, renal-intestinal or renal-peritoneal reflexes. As a rule, congenital malformations of the urinary tract are important because of 'their complications since otherwise they cause little or no discomfort. The above case was admitted to the hospital as a result of a road accident, and not because of complaints due to kidney disorder.

It remains only to stress the importance of a complete investigation in every case of hypertension in the young because of the beneficial effects of nephrectomy in a good number of cases of unilateral pyelonephritic contracted kidney.

Indications for nephrectomy. The following are the usually accepted criteria for removal of a diseased kidney :- (a) The blood pressure must be persistently high and of recent origin (two years). (b) The renal lesion must be confined to one kidney with impairment in the function of that kidney. (c) The combined renal function of both the kidneys must be within normal limits. (d) Ophthalmic changes are generally considered as contra-indications but the case quoted above of Kennedy. (I94I, I945) showed angio-spastic retinitis which cleared up after nephrectomy.

\section{Summary}

The pathogenesis of congenital megaloureter with ascending pyelonephritis, the causes of renal ischaemia and the role of renal ischaemia in the production of hypertension are reviewed.

A case of congenital megalo-ureter and pyelonephritis in, a boy aged I 3 years is described. Blood pressure was restored to normal after nephro-ureterectomy.

I wish to express my thanks to Dr. F. J. Power, Medical Superintendent, for permission to publish this case.

I also wish to thank Mr. J. Carver and Mr. GlynThomas for their surgical assistance, and Dr. N. Nothmann for his criticism.

\section{BIBLIOGRAPHY}

GRAEFI and PAGE, H. (1940), Amer. Fourn. Path., 16, 211. BUTLER, A. M. (1937), Fourn. of Clinical Investigation, 16, 889. KENNEDY, R. L. J., BARKER, N. W. and WALTERS, W. (194I), Amer. Fourn. of Diseases of Children, 61, I.

KENNEDY, R. L. J., BARKER, N. W. and WALTERS, W. (1945), Amer. Fourn. of Diseases of Children, 63, 3. BOYD, W. (1942), Fourn. of the Canadian Med. Ass., 47, 2. ROBERTSON, J. (1946), Proceedings of the Royal Society of Medicine, 39, II.

CHAWALLA, R. (1927), Urol. and Cutan. Rev., 3I.

GOLDBLATT, H., LYNCH, J., HANZEL, R. F., SUMMERVILLE, W. W. (I934), $\mathcal{F}$. Exp. Med., 59, 347.

SAPHIR, O. and BELLINGER, J. (1940), Arch. Int. Med., 66, $54 \mathrm{I}$.

GRAJEWSKI, L. E. (1940), $\mathcal{F}$. of Urology, 44, r.

BENJAMIN, S. (1941), Abeshouse Surgery, ro, I. 